\title{
Study on AHRS Sensor for Unmanned Underwater Vehicle ${ }^{\dagger}$
}

\author{
Ho-Sung Kim ${ }^{1}$, Hyeung-Sik Choi ${ }^{2 *}$, Jong-su Yoon ${ }^{2}$ and P.I. Ro ${ }^{3}$ \\ ${ }^{1}$ Ocean System Engineering, Samsung Tales, Samsung Tales, Kyungbook Gumi-city, Korea \\ ${ }^{2}$ Department of Mechanical and Energy System Engineering, Busan, Korea Maritime University, Korea \\ ${ }^{3}$ Department of Mechanical and Aerospace Engineering, North Carolina State University, Raleigh, NC27607, USA
}

(Manuscript Received June 9, 2011; Revised July 5, 2011; Accepted July 27, 2011)

\begin{abstract}
In this paper, for the accurate estimation of the position and orientation of the UUV (unmanned underwater vehicle), an AHRS (Attitude Heading Reference System) was developed using the IMU (inertial measurement unit) sensor which provides information on acceleration and orientation in the object coordinate and the initial alignment algorithm and the E-KF (extended Kalman Filter). The initial position and orientation of the UUV are estimated using the initial alignment algorithm with 3-axis acceleration and geomagnetic information of the IMU sensor. The position and orientation of the UUV are estimated using the AHRS composed of 3-axis acceleration, velocity, and geomagnetic information and the E-KF. For the performance test of the orientation estimation of the AHRS, a testbed using IMU sensor(ADIS16405) and DSP28335 coded with an E-KF algorithm was developed and its performance was verified through tests.
\end{abstract}

Keywords: AHRS Sensor, Extended Kalman filter, UUV(Unmanned Underwater Vehicle)

\section{Introduction}

An unmanned underwater vehicle (UUV) needs an ultrasonic observation system such as LBL (Long baseline), SBL (Short baseline), and USBL (Ultra short baseline) etc. since electric waves are not able to pass through water [1]. However, although the position error of the ultrasonic sensor does not diverse as time lapses, due to the position error of high frequency and the characteristics of ultrasonic waves inside the water, it has a long sampling frequency. Also, sensors such as DVL (Doppler velocity logger) or IMU (inertial measurement unit) have accumulative position errors though they have a relatively fast sampling time. . For these reasons, a complex navigation system

\footnotetext{
${ }^{*}$ Corresponding author. Tel.: +82-54-460-8705, Fax.: +82-54-460-8519. E-mail address: hosung0608.kim@samsung.com. Copyright $@$ KSOE 2011.
}

composed of DVL, IMU, USBL, GPS sensors is required[2][3][4][5].

In this paper, for the accurate estimation of the position and orientation of the UUV, the AHRS (Attitude Heading Reference System) was developed using the IMU sensor which provides information on acceleration and orientation in the object coordinate and the initial alignment algorithm. The initial position and orientation of the UUV are estimated using the initial alignment algorithm with 3-axis acceleration and geomagnetic information of the IMU sensor. The initial alignment in AHRS by which the orientation of the body is calculated between the navigation coordinate and body coordinate refers to the calculation of DCM (Direction Cosine matrix) which expresses the alignment of the body before entering the navigation mode. A Kalman filter, which is used mostly in initial alignment, requires a long calculation and execu- 
tion time. In the initial alignment which is proposed in this paper, the algorithm was simplified by using only the sensor outputs of three axis acceleration and three axis geomagnetism, and the estimated time for initial orientation was reduced. In the navigation mode, by using the Extended Kalman filter (EKF) with the orientation value of the initial alignment, the orientation value of the UUV is estimated. The measured values of the EKF are constituted with nine data of three axis angular velocities, gravity, and a geomagnetism sensor, and the estimated values are constituted with three axis angular velocities and four quaternion elements.

\section{Initial alignment of sensor}

The navigation coordinate is defined as the local horizontal coordinate of which the origin is the center of mass and the other three axes face toward north, east, and the vertical lower side, and the Daxis is perpendicular to the earth's ellipsoid, the Eaxis points directly east, and the $\mathrm{N}$-axis points directly north as shown in Fig. 1.

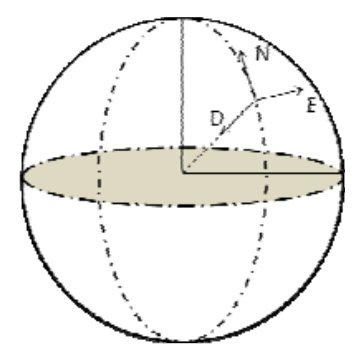

Fig. 1. Navigation Coordinate of AUV

A body coordinate is a coordinate in which the center resides at the center of mass in the UUV and it is defined such that its Z-axis is the Roll, its $\mathrm{Y}$ axis is the Pitch, and its $\mathrm{Z}$-axis is the Yaw of the AUV as shown in Fig. 2.

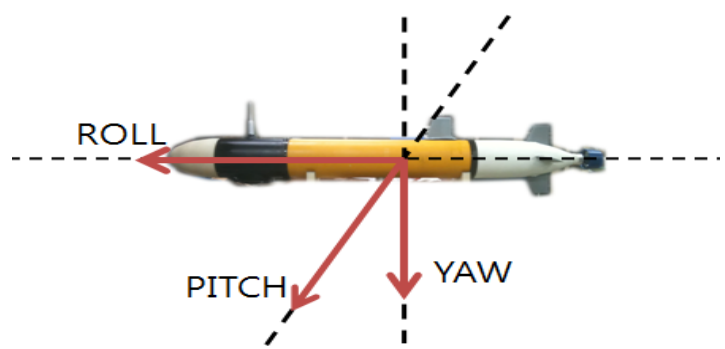

\subsection{Intial alignment algorithm}

To obtain the Roll $(\theta)$ and Pitch $(\theta)$ values, an assumption is required such that when the AUV is not moving, in the navigation coordinate, if the gravitational acceleration is multiplied by the matrix which transforms the navigation coordinate into the body coordinate, it becomes the acceleration output of the sensor, and it is expressed as the correlation equation Eq. (1). $\mathrm{C}_{\mathrm{n}}^{\mathrm{b}}$ is the transformation matrix from the navigation coordinate to the body coordinate and $\mathrm{a}_{\mathrm{x}}, \mathrm{a}_{\mathrm{y}}$, and $\mathrm{a}_{\mathrm{z}}$ are the acceleration sensor output values. $g$ represents gravity.

Because the three-axis angular velocity sensor used in the study is not equipped with a function to measure the angular velocity of earth rotation, an initial alignment using the geomagnetism sensor and the acceleration sensor was executed. In this paper, by using the output of the accelerometer in which gravitational acceleration information during initial alignment was included, the roll and pitch angles were calculated. Then, the yaw angle was calculated using the 3-axis geomagnetic sensor in which the calculated roll, pitch angles, and the vector value of geomagnetism are included. To calculate the roll and pitch angles, assuming that the AUV is not moving, if the gravity value is multiplied by DCM which transforms the navigation coordinate to the body coordinate, the obtained angle value, which would be the same as the output acceleration value is required, and it can be expressed as in Eq. (1).

$$
\left(\begin{array}{l}
a_{x} \\
a_{y} \\
a_{z}
\end{array}\right)=C_{n}^{b}\left(\begin{array}{l}
0 \\
0 \\
g
\end{array}\right)=\left(\begin{array}{l}
-g s_{\theta} \\
g c_{\theta} s_{\phi} \\
g c_{\phi} c_{\theta}
\end{array}\right)
$$

Where, $a_{x}, a_{y}$, and $a_{z}$ are output values of the inertial sensor and these are the vector values in the body coordinate. By calculating Eq. (1), roll and pitch angles are obtained as in Eq. (2).

$$
\begin{aligned}
& \phi_{\mathrm{n}}^{b}=\phi_{0}(\Phi) \phi_{2}(\theta) \phi_{\mathrm{n}}^{2}(\psi)
\end{aligned}
$$

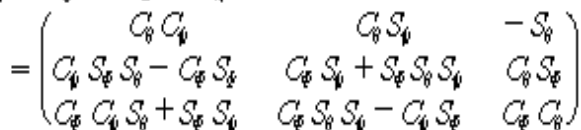

Fig. 2. Body Coordinate of AUV 


\subsection{Yaw Algorithm}

As shown in Eq.(1), multiplication of the gravity vector $g$ by the matrix which transforms the navigation coordinate into the disappearance of the yaw angle values. For this reason, another sensor other than acceleration is required. To solve this problem, an initial alignment using the geomagnetism sensor and acceleration sensor was executed.

When the $\mathrm{x}$-axis of the geomagnetic vector of earth in the navigation coordinate is defined as $m_{1}$, the $y$ axis as $m_{2}$, and the $\mathrm{z}$ axis as $m_{3}$, and the output of the geomagnetic sensor in the body coordinate is defined as the $x$ axis is $m_{x}$, the $y$ axis is $m_{y}$, and the $\mathrm{z}$ axis is $m_{z}$, and if these geomagnetic vectors are multiplied by the transformation matrix from the navigation coordinate to the body coordinate, we can assume these values as in Eq. (3).

$$
\left(\begin{array}{l}
m_{x} \\
m_{y} \\
m_{z}
\end{array}\right)=C_{n}^{b}\left(\begin{array}{l}
m_{1} \\
m_{2} \\
m_{3}
\end{array}\right)
$$

For the convenience of calculation to obtain the yaw angle, if $C_{n}^{b}$ is divided by the matrix about the yaw, roll, and pitch angles, respectively, the equation becomes Since $C_{n}^{b}=C_{1} C_{2}$, Eq. (4)

$C_{1}=\left(\begin{array}{ccc}c_{\theta} & 0 & -s_{\theta} \\ S_{\phi} s_{\theta} & c_{\phi} & S_{\phi} C_{\theta} \\ C_{\phi} s_{\theta} & -S_{\phi} & c_{\phi} C_{\theta}\end{array}\right), C_{2}=\left(\begin{array}{ccc}C_{\psi} & S_{\psi} & 0 \\ -S_{\psi} & C_{\psi} & 0 \\ 0 & 0 & 1\end{array}\right)$

Can be expressed as Eq. (5) below.

$$
\left(\begin{array}{l}
m_{x} \\
m_{y} \\
m_{z}
\end{array}\right)=C_{1} C_{2}\left(\begin{array}{l}
m_{1} \\
m_{2} \\
m_{3}
\end{array}\right)
$$

Where the inverse matrix of $C_{1}$ is multiplied to both sides of Eq.(5), which can be expressed as

$$
\begin{aligned}
& t_{3}=\frac{s_{\psi}}{c_{\psi}}=\frac{-m_{2} X+m_{1} Y}{-m_{1} X-m_{2} Y}=\tan (\psi) \\
& \psi=\tan ^{-1}\left(\frac{-m_{2} X+m_{1} Y}{-m_{1} X-m_{2} Y}\right) \\
& =\tan ^{-1}\left(\frac{-m_{2}\left(c_{\theta} m_{x}+s_{\phi} s_{\theta} m_{y}+c_{\phi} s_{\theta} m_{z}\right)+m_{1}\left(c_{\phi} m_{y}-s_{\phi} m_{z}\right)}{-m_{1}\left(c_{\theta} m_{x}+s_{\phi} s_{\theta} m_{y}+c_{\phi} s_{\theta} m_{z}\right)-m_{2}\left(c_{\phi} m_{y}-s_{\phi} m_{z}\right)}\right) \\
& =\tan ^{-1}\left(\frac{-m_{2} c_{\theta} m_{x}-m_{2} s_{\phi} s_{\theta} m_{y}-m_{2} c_{\phi} s_{\theta} m_{z}+m_{1} c_{\phi} m_{y}-m_{1} s_{\phi} m_{z}}{-m_{1} c_{\theta} m_{x}-m_{1} s_{\phi} s_{\theta} m_{y}-m_{1} c_{\phi} s_{\theta} m_{z}-m_{2} c_{\phi} m_{y}+m_{2} s_{\phi} m_{z}}\right) \\
& c_{q} m_{X}+s_{Y} s_{g} m_{\mathrm{W}}+c_{T} s_{q} m_{c}=c_{M} m_{1}+s_{\Psi} m_{2},
\end{aligned}
$$

Here, $C_{\mathrm{q}} m_{\mathrm{v}}-S_{\mathrm{W}} m_{\mathrm{c}}=-S_{\mathrm{W}} m_{1}+C_{\mathrm{W}} m_{2}$ ,then

for the convenience of calculation, if the substitution is made as $c_{\theta} m_{x}+s_{\phi} s_{\theta} m_{y}+c_{\phi} s_{\theta} m_{z}=X, c_{\phi} m_{y}-s_{\phi} m_{z}=Y$ , Eq. (8) is simplified as

$$
\begin{aligned}
& c_{\psi} m_{1}+s_{\psi} m_{2}=X \\
& -s_{\psi} m_{1}+c_{\psi} m_{2}=Y
\end{aligned}
$$

Where Eq. (8) can be expressed as a matrix form as Eq. (9)

$$
\left(\begin{array}{cc}
m_{1} & m_{2} \\
m_{2} & -m_{1}
\end{array}\right)\left(\begin{array}{l}
c_{\psi} \\
s_{\psi}
\end{array}\right)=\left(\begin{array}{l}
X \\
Y
\end{array}\right)
$$

Using Eq.(9), the yaw angle can be expressed as a closed-form as

$$
\begin{aligned}
& C_{1}^{-1}\left(\begin{array}{l}
m_{x} \\
m_{y} \\
m_{z}
\end{array}\right)=C_{2}\left(\begin{array}{l}
m_{1} \\
m_{2} \\
m_{3}
\end{array}\right) \\
& \left(\begin{array}{ccc}
c_{\theta} & s_{\phi} s_{\theta} & c_{\phi} s_{\theta} \\
0 & c_{\phi} & -s_{\phi} \\
-s_{\theta} & s_{\phi} s_{\theta} & c_{\phi} c_{\theta}
\end{array}\right)\left(\begin{array}{l}
m_{x} \\
m_{y} \\
m_{z}
\end{array}\right)=\left(\begin{array}{ccc}
c_{\psi} & s_{\psi} & 0 \\
-s_{\psi} & c_{\psi} & 0 \\
0 & 0 & 1
\end{array}\right)\left(\begin{array}{l}
m_{1} \\
m_{2} \\
m_{3}
\end{array}\right) \\
& \left(\begin{array}{c}
c_{\theta} m_{x}+s_{\phi} s_{\theta} m_{y}+c_{\phi} s_{\theta} m_{z} \\
c_{\phi} m_{y}-s_{\phi} m_{z} \\
-s_{\theta} m_{x}+s_{1} s_{\theta} m_{y}+c_{1} c_{\theta} m_{z}
\end{array}\right)=\left(\begin{array}{c}
c_{\psi} m_{1}+s_{\psi} m_{2} \\
-s_{\psi} m_{1}+c_{\psi} m_{2} \\
m_{3}
\end{array}\right)
\end{aligned}
$$

When the initial alignment for the roll and pitch angles is executed, since the value at the right side in Eq. (10) becomes a constant, the yaw angle can be obtained. 


\section{Coordinate transformation algorithm for} AHRS

The AHRS which expresses the attitude information and rotational angular information of the AUV in motion was constructed by implementing a coordinate transformation algorithm at the IMU sensor which is a body coordinate sensor. For this, EKF was applied, of which the initial value was taken from the initial alignment data.

The EKF is composed of a measurement matrix which is external input, an estimate matrix which is the final output, an F matrix which is the system model, and an $\mathrm{H}$ matrix. Also, the EKF process is divided into the estimation and assumption processes. In the estimation process, the current estimate matrix and error covariance are calculated based on the previous estimate matrix and error covariance. The estimate matrix used in the estimation process and system model $\mathrm{F}$ matrix is expressed as Eq. (11) and (12), respectively. Here, the estimate matrix comprises four quaternion elements and 3-axis angular velocity values.

$$
\begin{aligned}
x & =\left(G_{1} G_{1} G_{2} G_{3} W_{k} W_{d} W_{d}\right)^{T} \\
F & =\left(\begin{array}{ll}
R_{d \times 4} & O_{3 \times 3} \\
0_{3 \times 4} & I_{3 \times 3}
\end{array}\right)
\end{aligned}
$$

Here, the $R$ matrix is the orientation transformation matrix. The F matrix in Equation (10) comprises the matrix which connects the Estimate matrix of the previous condition to the Estimate matrix of the current condition. During the estimate process, after receiving input from the Estimate matrix during the estimation process, error covariance, and Measurement matrix and by using the $\mathrm{H}$ matrix in the system model, the final Estimate matrix and error covariance were calculated. The measurement matrix and $\mathrm{H}$ matrix which were used during the estimation process are the same as Equation (13) and Equation (14).

$$
z=\left(w_{\boldsymbol{k}} w_{\mathrm{g}} w_{\mathrm{A}} a_{\boldsymbol{k}} a_{\mathrm{g}} a_{\mathrm{A}} m_{\boldsymbol{k}} m_{\mathrm{g}} m_{\mathrm{A}}\right)^{T}
$$

The $\mathrm{H}$ matrix in Equation (11) is composed of the matrix which updates the estimate matrix as the measurement matrix.

$$
\mathrm{H}=\left[\begin{array}{ccccccc}
0 & 0 & 0 & 0 & 1 & 0 & 0 \\
0 & 0 & 0 & 0 & 0 & 1 & 0 \\
0 & 0 & 0 & 0 & 0 & 0 & 1 \\
\frac{\partial \mathrm{h}_{1}}{\partial \mathrm{q}_{0}} & \frac{\partial \mathrm{h}_{1}}{\partial \mathrm{q}_{1}} & \frac{\partial \mathrm{h}_{1}}{\partial \mathrm{q}_{2}} & \frac{\partial \mathrm{h}_{1}}{\partial \mathrm{q}_{2}} & 0 & 0 & 0 \\
\frac{\partial \mathrm{h}_{2}}{\partial \mathrm{q}_{0}} & \frac{\partial \mathrm{h}_{2}}{\partial \mathrm{q}_{1}} & \frac{\partial \mathrm{h}_{2}}{\partial \mathrm{q}_{2}} & \frac{\partial \mathrm{h}_{2}}{\partial \mathrm{q}_{2}} & 0 & 0 & 0 \\
\frac{\partial \mathrm{h}_{3}}{\partial \mathrm{q}_{0}} & \frac{\partial \mathrm{h}_{3}}{\partial \mathrm{q}_{1}} & \frac{\partial \mathrm{h}_{2}}{\partial \mathrm{q}_{2}} & \frac{\partial \mathrm{h}_{3}}{\partial \mathrm{q}_{2}} & 0 & 0 & 0 \\
\frac{\partial \mathrm{h}_{4}}{\partial \mathrm{q}_{0}} & \frac{\partial \mathrm{h}_{4}}{\partial \mathrm{q}_{1}} & \frac{\partial \mathrm{h}_{4}}{\partial \mathrm{q}_{2}} & \frac{\partial \mathrm{h}_{4}}{\partial \mathrm{q}_{2}} & 0 & 0 & 0 \\
\frac{\partial \mathrm{h}_{5}}{\partial \mathrm{q}_{0}} & \frac{\partial \mathrm{h}_{5}}{\partial \mathrm{q}_{1}} & \frac{\partial \mathrm{h}_{5}}{\partial \mathrm{q}_{2}} & \frac{\partial \mathrm{h}_{5}}{\partial \mathrm{q}_{3}} & 0 & 0 & 0 \\
\frac{\partial \mathrm{h}_{6}}{\partial \mathrm{q}_{0}} & \frac{\partial \mathrm{h}_{6}}{\partial \mathrm{q}_{1}} & \frac{\partial \mathrm{h}_{6}}{\partial \mathrm{q}_{2}} & \frac{\partial \mathrm{h}_{6}}{\partial \mathrm{q}_{3}} & 0 & 0 & 0
\end{array}\right]
$$

Using Equation (14) through Equation (1) and fusing the angular velocity sensor, and geomagnetic sensor, the AHRS sensor appropriate to navigation is composed. A simulation and performance test of the developed AHRS has been carried out to verify good performance.

\section{Simulation and Performance test}

\subsection{Simulation}

For the performance test of the AHRS algorithm, simulink in Matlab has been used. As an input value for the simulation program, $10 N$ of the $x, y$, and $z$ axes and force as well as the moment force which has a sine wave form with $0.69 \mathrm{Nm}$ toward the $\mathrm{x}$ axis, $0.66 \mathrm{Nm}$ toward the $y$-axis, and $0.36 \mathrm{Nm}$ toward the $z$ - axis were used. For angular velocity, acceleration, and the geomagnetism sensor, a modeled sensor of which the average noise level was 0 and the variance was 2.54E-4, 8.0E1 was used. Fig. 3 shows simulation results where the top graph represents the estimate value using EKF, and the middle graph indicates the actual value.

The lowest graph shows the simultaneous expression of estimates and actual values. As shown in the third graph, the estimated value using EKF is quite close to the actual values. 

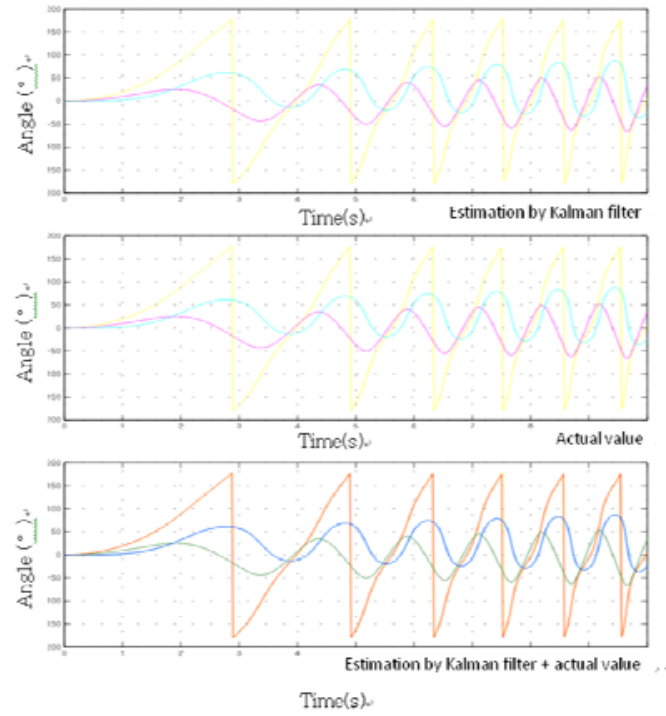

Fig. 3. Simulation results

\subsection{Test result}

For the implementation of the initial alignment and AHRS algorithm to the actual USV, a hardware system using a DSP28335 microprocessor and a ADIS16405 IMU sensor was constituted as shown Fig. 4. Here, DSP28335 has a $150 \mathrm{MHz}$ speed and a floating point calculation performance. The specification of ADIS16405 is shown in Table 1. The signal processing sensed from the IMU sensor was estimated in real time using the algorithm of EKF shown in Fig. 5 [6-9].

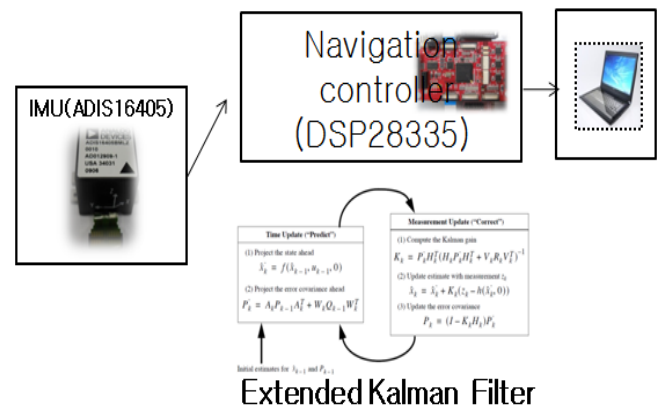

Fig. 4. IMU estimation system using E-Kalman

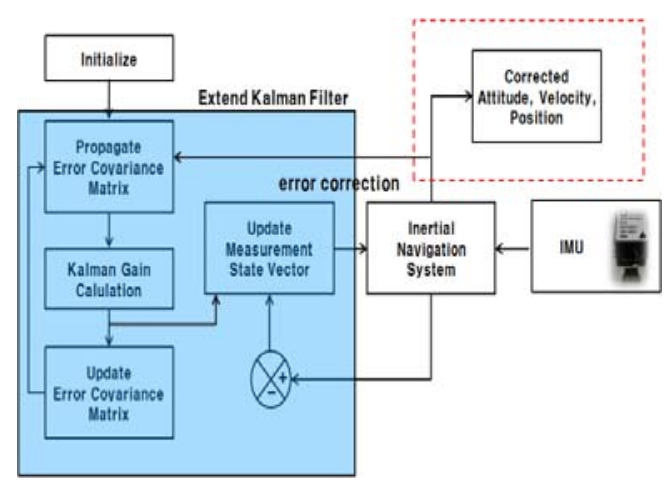

Fig. 5. IMU estimation process using E-Kalman

Table 1. ADIS16405 Specifications

\begin{tabular}{|c|c|c|}
\hline \multirow{3}{*}{ Output } & \multicolumn{2}{|c|}{3 axis angular velocity } \\
\hline & \multicolumn{2}{|c|}{3 axis acceleration } \\
\hline & \multicolumn{2}{|c|}{3 axis geomagnetism } \\
\hline \multirow{3}{*}{ Specifications } & $\begin{array}{l}3 \text { axis angular } \\
\text { velocity noise }\end{array}$ & $0.9^{\circ}$ \\
\hline & $\begin{array}{l}3 \text { axis accelera- } \\
\text { tion noise }\end{array}$ & $9 \mathrm{mg}$ \\
\hline & $\begin{array}{l}3 \text { axis geomag- } \\
\text { netism noise }\end{array}$ & $\begin{array}{c}1.25 \\
\text { mgauss }\end{array}$ \\
\hline
\end{tabular}

Two performance tests were carried out: one involves testing the estimate ability of the roll, pitch, and yaw angles of the developed AHRS under stationary condition. The other test involves testing the estimate ability of the AHRS in motion. For this, the AHRS fixed on a plate was rotated at angle ranges of $0^{\circ}-100^{\circ}$. Through tests, it has been confirmed that the Gimbal lock phenomenon did not occur and the orientation angles could be estimated within the hardware errors with less noise. Figs. $6 \sim 8$ show the test results of the three angles of the IMU sensor on the stationary plate. From the figure, it is shown that.

The estimates of the roll angle have an error range of $0.22^{\circ}$, the estimates of the pitch angle have an error range of $0.24^{\circ}$, and the estimates of the yaw angle have an error range of $0.21^{\circ}$. Fig. 9 shows the test results of rotating the AHRS sensor from $0^{\circ}$ to $100^{\circ}$. It could be confirmed that within these angles, the Gimbal lock phenomenon did not occur and the orientation angles could be estimated within the hardware. 


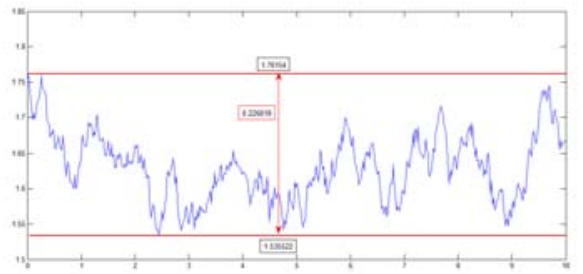

Fig. 6. Roll test result under stationary condition

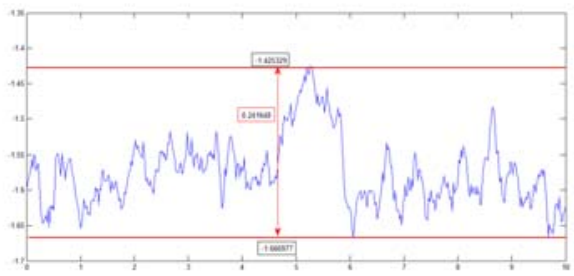

Fig. 7. Pitch test result under stationary condition

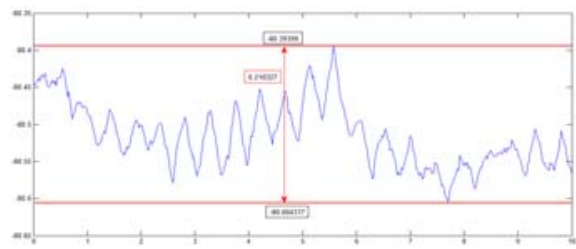

Fig. 8. Yaw test result under stationary condition

\section{Conclusion}

For the motion estimation of the AUV, an initial alignment method has been designed in which the 3axis acceleration and geomagnetism information of an IMU sensor were used and the motion has been estimated by the construction of an AHRS after fusing 3-axis angular velocity, acceleration, and geomagnetism data. For the performance test of the motion estimation in AHRS for the AUV, a test apparatus has been constructed using ADIS16405, which is an IMU sensor and DSP28335, on which the signal processing algorithm and an EKF algorithm were transplanted and its performance was tested.

According to the test, the estimates of the roll angle have an error range of $0.22^{\circ}$, the estimates of the pitch angle have an error range of $0.24^{\circ}$, and the estimates of the yaw angle have an error range of $0.21^{\circ}$. This means that the output angles of the roll, pitch, and yaw in the developed AHRS have less angle errors than the 3-axis angular velocity noise of the specified ADIS16405 IMU sensor in Table 1.

\section{Acknowledgement:}

This research was a part of the project titled "Development of Key Marine Equipments for Enhancement of Ocean Industry - Development of Underwater Manipulator and Thrusting System Driven by Electric Motor" funded by the Ministry of Land, Transport and Maritime Affairs, Korea.

\section{Reference}

[1] Milne, P. H., Underwater acoustic positioning systems, Gulf Publishing Company, (1983).

[2] Woolven, S. and Field, M., POS/SV - an aided intertial navigation system for submersible vehicles, Proc. Of Oceans '98 Conference, Vol. 1, (1998) pp. 103 107,

[3] Yun, X., Bachmann, E. R., McGhee, R.B., Whalen R. H., Roberts, R. L., Knapp R. G., Healey, A. J. and Zyda, M. J., Testing and evaluation of an intergrated GPS/INS system for small AUV navigation, IEEE J. of Oceanic Engineering, Vol. 24, No.3, (1999) pp. 396 404.

[4] An, P. E., Healey, A. J., Smith, S. M. and Dunn, S.E., New experimental results on GPS/INS navigation for Ocean Voyager 2 AUV, Proceedings of AUV '96, 1996 pp. 249 255.

[5] Marco, D. B. and Healey, A. J., Command, control, and navigation experimental results with the NPS ARIES AUV, IEEE j. of Oceanic Engineering, Vol. 26 No. 4, (2001) pp. 466 476.

[6] Ppoulis, Probability, Random Variables and Stochastic Process, Mc-Graw Hill.

[7] Maybeck, Peter S., Stochastic Models, Estimation, and Control, Vol.1.

[8] Alberto Leon-Garcia, Probability and Random Process for Electrical.

[9] Patrick Billingsley, Probability and Measure, Ed. Wiley, (1979). 